\title{
Genetic risk stratification, pivotal to precluding the CAD pandemic
}

\author{
Robert Roberts \\ Department of Medicine, Dignity Health at St. Joseph's Hospital and Medical Center, Phoenix, AZ 85013, USA. \\ Correspondence to: Prof. Robert Roberts, Department of Medicine, Dignity Health at St. Joseph's Hospital and Medical Center, \\ 500 W Thomas Rd Ste 480, Phoenix, AZ 85013, USA. E-mail: robert.roberts@dignityhealth.org
}

How to cite this article: Roberts R. Genetic risk stratification, pivotal to precluding the CAD pandemic. J Cardiovasc Aging 2021;1:4. https://dx.doi.org/10.20517/jca.2021.05

Received: 21 May 2021 Accepted: 31 May 2021 Available online: 6 Jun 2021

Academic Editor: AJ Marian Copy Editor: Yue-Yue Zhang Production Editor: Yue-Yue Zhang

A major cardiovascular trend has been the development of Preventive Cardiology. In the past 10 years, Coronary Artery Disease (CAD) has become pandemic, being the number one killer in low, middle, and high-income countries. Epidemiologists identified the conventional risk factors predisposing to CAD more than 50 years ago but were aware even then of an additional genetic predisposition. Technology was not available until recently to pursue the DNA sequences mediating this risk. Today, the predisposition for common diseases such as CAD is claimed to be $50 \%$ genetic and $50 \%$ acquired. Early primary prevention of $\mathrm{CAD}$ based on genetic risk stratification has the potential to transform our approach to this disease.

\section{DISCOVERY OF GENETIC RISK VARIANTS PREDISPOSING TO CAD}

Unlike single-gene disorders, the phenotype of common disorders such as CAD is due to multiple genes interacting with multiple acquired factors. Genetic linkage analysis of pedigree's provided the golden era for discovering genes responsible for single-gene disorders but was inappropriate for polygenic disorders. Recent scientific contributions predominantly, the Human Genome and HapMap Projects, enabled us to pursue the discovery of the DNA variants contributing to polygenic disorders. HapMap Project mapped and annotated millions of single nucleotide polymorphisms (SNPs) which provided the DNA markers necessary to pursue genome-wide association studies (GWAS). The human DNA sequence is $99 \%$ identical with the remaining $1 \%$ being unique sequences responsible for the individual distinguishing features. Included within the $1 \%$ are SNPs of which the number has remained fairly constant at about 5 million per 
genome. Stranger et al. ${ }^{[1]}$ claim that $80 \%$ of individual human attributes are due to these SNPs. Utilizing GWAS and SNPs as markers, the first genetic risk variant for $\mathrm{CAD}^{[2,3]}$ was discovered on the short arm (p) of chromosome 9 (9p21). The formation of international consortia led by CARDIoGRAMplusC4D enabled sample sizes of hundreds of thousands leading to the discovery of hundreds of genetic risk variants predisposing to $\mathrm{CAD}^{[4,5]}$. Each genetic risk variant imparts minimal increased risk for CAD with over $80 \%$ occurring in non-protein-coding regions of the genome. Thus, the risk is mediated by increasing or decreasing the expression of protein-coding genes. The mechanism mediating the risk for CAD is unknown for over half of the genetic risk variants. The genetic risk for CAD is proportional to the number of genetic risk variants inherited, so can be expressed as a single number referred to as a Polygenic Risk Score (PRS).

\section{CONVENTIONAL RISK FACTORS FOR CAD ARE LESS THAN ADEQUATE TO STRATIFY FOR PRIMARY PREVENTION}

A major interest is whether the PRS would effectively improve risk stratification for CAD. Secondary prevention of the clinical sequelae of $\mathrm{CAD}$ has been very effective. Primary prevention is also effective but limited due to a lack of means to detect those at increased risk. It is claimed 50\% of individuals will experience at least 1 cardiac event in their lifetime. Secondly, CAD, which starts early in life, develops gradually and is more effectively prevented by early prevention. The conventional risk factors are agedependent and often not increased to provide risk stratification until the fifth or sixth decade. Plasma cholesterol, a primary causative factor for CAD, also increases with age but already exceeds the recommended level of $70 \mathrm{mg} / \mathrm{dL}$ in men and women by the fourth decade ${ }^{[6]}$. Short of treating everyone with increased plasma cholesterol, knowing 50\% will not benefit but be exposed to the same side effects and cost, why not risk stratify using the PRS. Risk due to one's DNA is determined at the time of conception and does not change in one's lifetime. This enables one to determine the risk for CAD any time after birth and initiate early prevention. This will provoke at least two questions. How effective is the PRS in stratifying for CAD risk? Does genetic risk respond to treatment?

\section{PRS IS SUPERIOR TO CONVENTIONAL RISK FACTORS FOR RISK STRATIFICATION}

The initial evaluation of the CAD PRS in $2012^{[7]}$ utilizing only 12 genetic risk variants showed only slight improvement. The increased number of genetic risk variants led to their encryption into microarrays enabling stratification of large sample sizes. Abraham et al.$^{[8]}$ genotyped 16,082 individuals utilizing 49,310 risk variants derived from CARDIoGRAMplusC4D. The group with the highest genetic risk score was associated with the most cardiac events. Inouye et al. ${ }^{[9]}$ genotyped 500,000 individuals from the UK biobank with a microarray having 1.7 million genetic risk variants and categorized their results into low, intermediate, and high risk. The top $20 \%$ with the highest PRS had a 4 -fold increased risk for CAD. A similar analysis by Khera et al. ${ }^{[10]}$ utilizing a sample size of 388,978 individuals was genotyped with a microarray containing 6.6 million risk variants for CAD. They observed the top $8 \%$ with the highest PRS had a 3 -fold increased risk for CAD and the top 0.5\%, a 5-fold increased risk for CAD. Analysis showed conventional risk factors would not have detected most of the individuals at risk. In the group with the highest PRS, only $20 \%$ had hypercholesterolemia, $35 \%$ of family history, and $28 \%$ with hypertension. All of these studies showed the PRS to be relatively independent of conventional risk factors, including family history.

\section{CHOLESTEROL-LOWERING DRUGS SIGNIFICANTLY REDUCE THE GENETIC RISK FOR CAD}

To further assess the power of PRS, retrospective genotyping of large clinical trials was performed. Mega et al. ${ }^{[11]}$ used 27 genetic risk variants in a sample size of 48,421 consisting of 4 randomized placebocontrolled trials to assess the effect of statins on cardiac events. Two of the trials were primary prevention 
JUPITER (Justification for the Use of Statins in Prevention: an Intervention Trial Evaluating Rosuvastatin) and ASCOT (Anglo-Scandinavian Cardiac Outcomes Trial), and the other two secondary prevention CARE (Cholesterol and Recurrent Events), and PROVE-IT-TIMI. Pravastatin or Atorvastatin Evaluation and Infection Therapy-Thrombolysis in Myocardial Infarction. Individuals with the highest PRS received the most benefit from statin therapy. The increased power of the PRS over conventional risk factors was evident from the number needed to treat to prevent one cardiac event was decreased 3 -fold from that of conventional risk factors. Genetic Risk stratification was equally effective in primary and secondary prevention. Another large clinical trial ${ }^{[12]}$ referred to as the West of Scotland Coronary Prevention Study was genotyped and the PRS was based on 57 genetic risk variants. Statin therapy in the high genetic risk group was associated with a $44 \%$ reduction of cardiac events, $v s$. only $24 \%$ in the intermediate and low-risk genetic groups. The number needed to treat to prevent one cardiac event in the high genetic risk group was 13.

Genetic stratification for risk of CAD was performed using the PRS in two recently randomized placebocontrolled clinical trials Fourier (Further Cardiovascular Outcomes Research with PCSK9 in subjects with Elevated Risk) ${ }^{[13]}$ and ODYSSEY (Evaluation of Cardiovascular outcomes after an acute coronary syndrome during treatment with Alirocumab) ${ }^{[14]}$. Patients receiving Evolocumab therapy had a 13\% relative reduction in the group stratified by conventional risk factors without high genetic risk and a 31\% reduction in the high genetic risk group with or without conventional risk factors. The second trial (ODYSSEY) enrolled 11,953 individuals. Individuals with the highest PRS had the highest risk for CAD and Alirocumab was associated with a $37 \%$ reduction in cardiac events $v$ s. a $13 \%$ reduction in the group with the lowest PRS.

\section{FAVORABLE LIFESTYLE SIGNIFICANTLY REDUCES GENETIC RISK FOR CAD}

An analysis of four prospective cohorts by Khera et al. ${ }^{[15]}$ involved a sample size of 55,685 individuals. Comparing favorable with unfavorable lifestyle. Those with the highest PRS for CAD (top 20\%) had a 90\% higher risk of cardiac events than the remainder. The group with the highest PRS and a favorable lifestyle had $46 \%$ fewer cardiac events than those with an unfavorable lifestyle.

Utilizing a sample size of 468,095 individuals from the UK Biobank, Tikkanen et al.$^{[16]}$ performed genetic risk stratification and assessed the effect of physical activity on cardiac events. The physical activity was the handgrip for 3 seconds and cardiac respiratory fitness was assessed by oxygen consumption performed while exercising on a stationary bike. The group with the highest PRS exhibited $49 \%$ fewer cardiac events.

\section{PRS SHOULD BE INCLUDED IN THE CLINICAL GUIDELINES FOR CAD AS AN ENHANCER FOR RISK STRATIFICATION}

In an era where medicine is supposed to be more precise and cost-effective, one would prefer initiating primary prevention in those at greatest risk for the disease. The PRS has been evaluated as a risk stratifier for CAD in over 1 million individuals by several investigators and results show it to be superior to conventional risk factors. Secondly, the PRS is relatively independent of the conventional risk factors. Thirdly, the PRS is independent of age, given it is determined at conception and does not change in one's lifetime. Fourthly, the genetic risk has been shown to be reduced by favorable lifestyle changes and drugs that decrease plasma cholesterol. The PRS is not included in the guidelines but could be an enhancer for risk stratification, similar to the recent inclusion of the calcium score. Riveros-Mckay et al. ${ }^{[17]}$ compared the PRS to the United States guidelines and showed $10 \%$ of CAD cases were misclassified as low risk by PCE which were correctly classified as high risk by the integrated PRS. They up-classified 7\% of the population (7.4 million individuals) to high risk, qualifying them for statin prevention. The estimated cost of the PRS is in the range of $\$ 100-\$ 200$. The application of the PRS to males in their 20's and 30's or females in their 40 's would be expected to transform the primary prevention of the world's number one killer, CAD. 


\section{DECLARATIONS}

\section{Authors' contributions}

The author contributed solely to the article.

\section{Availability of data and materials \\ Not applicable.}

\section{Financial support and sponsorship}

None.

\section{Conflicts of interest}

The author declared that there are no conflicts of interest.

\section{Ethical approval and consent to participate \\ Not applicable.}

\section{Consent for publication}

Not applicable.

\section{Copyright}

(c) The Author(s) 2021.

\section{REFERENCES}

1. Stranger BE, Forrest MS, Dunning M, et al. Relative impact of nucleotide and copy number variation on gene expression phenotypes. Science 2007;315:848-53. DOI PubMed PMC

2. McPherson R, Pertsemlidis A, Kavaslar N, et al. A common allele on chromosome 9 associated with coronary heart disease. Science 2007;316:1488-91. DOI PubMed PMC

3. Helgadottir A, Thorleifsson G, Manolescu A, et al. A common variant on chromosome 9p21 affects the risk of myocardial infarction. Science 2007;316:1491-3. DOI PubMed

4. Assimes TL, Roberts R. Genetics: implications for prevention and management of coronary artery disease. J Am Coll Cardiol 2016;68:2797-818. DOI PubMed

5. Schunkert H, von Scheidt M, Kessler T, Stiller B, Zeng L, Vilne B. Genetics of coronary artery disease in the light of genome-wide association studies. Clin Res Cardiol 2018;107:2-9. DOI PubMed

6. Roberts R, Fair J. Clinical application of genetic prediction in the management of CAD. IJIRMS 2021;6:46-52.

7. Davies RW, Dandona S, Stewart AF, et al. Improved prediction of cardiovascular disease based on a panel of single nucleotide polymorphisms identified through genome-wide association studies. Circ Cardiovasc Genet 2010;3:468-74. DOI PubMed PMC

8. Abraham G, Havulinna AS, Bhalala OG, et al. Genomic prediction of coronary heart disease. Eur Heart J 2016;37:3267-78. DOI PubMed PMC

9. Inouye M, Abraham G, Nelson CP, et al. Genomic risk prediction of coronary artery disease in 480,000 adults. J Am Coll Cardiol 2018;72:1883-93. DOI PubMed PMC

10. Khera AV, Chaffin M, Aragam KG, et al. Genome-wide polygenic scores for common diseases identify individuals with risk equivalent to monogenic mutations. Nat Genet 2018;50:1219-24. DOI PubMed PMC

11. Mega JL, Stitziel NO, Smith JG, et al. Genetic risk, coronary heart disease events, and the clinical benefit of statin therapy: an analysis of primary and secondary prevention trials. Lancet 2015;385:2264-71. DOI PubMed PMC

12. Natarajan P, Young R, Stitziel NO, et al. Polygenic risk score identifies subgroup with higher burden of atherosclerosis and greater relative benefit from statin therapy in the primary prevention setting. Circulation 2017;135:2091-101. DOI PubMed PMC

13. Marston NA, Kamanu FK, Nordio F, et al. Predicting benefit from evolocumab therapy in patients with atherosclerotic disease using a genetic risk score. Circulation 2020;141:616-23. DOI PubMed PMC

14. Damask A, Steg PG, Schwartz GG, et al. Patients with high genome-wide polygenic risk scores for coronary artery disease may receive greater clinical benefit from alirocumab treatment in the ODYSSEY OUTCOMES trial. Circulation 2020;141:624-36. DOI PubMed

15. Khera AV, Emdin CA, Drake I, et al. Genetic risk, adherence to a healthy lifestyle, and coronary disease. $N$ Engl $J$ Med 2016;375:2349-58. DOI PubMed PMC

16. Tikkanen E, Gustafsson S, Ingelsson E. Associations of fitness, physical activity, strength, and genetic risk with cardiovascular disease: longitudinal analyses in the UK Biobank study. Circulation 2018;137:2583-91. DOI PubMed PMC

17. Riveros-Mckay F, Weale ME, Moore R, et al. Integrated polygenic tool substantially enhances coronary artery disease prediction. Circ 
Genom Precis Med 2021;14:e003304. DOI PubMed 